\title{
The Index of Scattering Operators of Dirac Equations
}

\author{
Taku Matsui \\ Research Institute for Mathematical Sciences, Kyoto University, Kyoto 606, Japan
}

\begin{abstract}
A new index formula of Atiyah Singer type for scattering operators is proved. The index corresponds to the vacuum polarization of the Fermion (on the Minkowski space) coupled to an external non abelian gauge field.
\end{abstract}

\section{Introduction}

Geometric features of gauge theories have been extensively investigated since the discovery of instantons. In spite of several successes, the methods have been based on the compactification of the space-time manifold, which is of great disadvantage if we wish to find precise relations between the obtained results and realistic quantum field theories. Even though the non-compactness of $\mathbb{R}^{4}$ is controlled by the boundary condition at infinity, we don't know how to relate results for compact Riemannian manifolds to the Minkowski space (the theory of elliptic operators, e.g. Atiyah-Singer's index theorem to the theory of hyperbolic differential operators). In the Minkowski space, we have no effective geometric tools for the study of anomalies and other topological effects.

The motivation of the present work is to find a geometric invariant of gauge theories in quantum systems on the Minkowski space. We explain the background of our results in more detail now.

Consider a (second) quantized charged Fermion coupled with an external field on a Fock space. Mathematically the Fermion field is an element of a CAR (canonical anticommunication relations) algebra which is isomorphic to an infinite dimensional Clifford algebra $\mathfrak{A}$ on a complex Hilbert space $\mathscr{H}$ with an antiunitary involution. The Fock representation is an irreducible representation of $\mathfrak{U}$ with a special vector $\Omega$ called vacuum. This representation is completely specified by a projection $P$ on $\mathscr{H}$. See [1].

In most physical situations, the representation $u(g)$ on $\mathscr{H}$ of a compact group $G$ is given which is canonically lifted to an action $\alpha_{g}$ of $G$ on $\mathfrak{A}$ via Bogoliubov automorphisms. $\alpha_{g}$ is identified with the global gauge transformation, and the fixed point subalgebra $\mathfrak{U}^{G}$ of this action is regarded as the observable algebra.

Address after April 1987: Department of Mathematics, Faculty of Sciences, Tokyo Metropolitan University, Setagaya-ku, Tokyo 158, Japan 
In the free Dirac field, the projection $P$ above is the positive spectral projection of the free Dirac operator and $G=U(1)=\{Z \in \mathbb{C},|Z|=1\}$, which distinguishes particles and antiparticles. If $[P, u(g)]=P u(g)-u(g) P=0$, the above $G$ action is unitarily implemented on the Fock space associated to $P$ by $W(g)$ satisfying

$$
W(g) \Omega=\Omega, \quad W(g) Q W(g)=\alpha_{g}(Q), \quad Q \in \mathfrak{A} .
$$

For the global $U(1)$ gauge transformation in the free Dirac field the infinitesimal generator of $W(g)$ is identified with the charge operator.

Given a gauge potential, the associated scattering operator $S$ of the Dirac equation defines a scattering automorphism $\alpha_{S}$ of $\mathfrak{U}$ via a Bogoliubov automorphism. $\alpha_{S}$ is unitarily implemented on the Fock space associated to $P$ if and only if $[P, S]$ is in the Hilbert-Schmidt class. If this condition is satisfied, the implementor $W_{S}$ for $\alpha_{S}$ is regarded as the second quantized scattering operator. In this framework, the in and out vacuua are identified with $\Omega$ and $W_{S} \Omega$ respectively,

$$
\Omega_{\text {in }}=\Omega, \quad \Omega_{\text {out }}=W_{S} \Omega .
$$

The charge defined by $W\left(e^{i \theta}\right)(G=U(1))$ is not necessarily conserved in the scattering, i.e. $\left[W\left(e^{i \theta}\right), W_{S}\right] \neq 0$ even though it is at the quantum mechanical level on $\mathscr{H}$. We can only show

$$
W\left(e^{i \theta}\right) \Omega_{\text {out }}=e^{\text {in } \theta} \Omega_{\text {out }} .
$$

The integer $n$ is the shift of charge of vacuum during the scattering. The appearance of non-trivial charge shift is referred to as vacuum polarization. The mathematical meaning of the phase factor in (1.3) is that the cyclic representation of the vector state $\Omega_{\text {out }}$ of the observable algebra $\mathfrak{H}^{G}$ is non-equivalent to that of $\Omega_{\text {in }}$ if $n \neq 0$.

By the result of [3], the charge shift is computed by

$$
n=\text { ind }_{P \mathscr{H}} P S P \text {, }
$$

where the right-hand side of (1.4) is the index of the operator PSP restricted to $P \mathscr{H}$. (Note that the Fredholm property of PSP is ensured by the compactness of $[P, S]$ and unitarity of $S$. See Lemma 3.9.) See also [7]. Thus we are led to the following question:

(A) Can we find potentials with scattering operators $S$ satisfying

(i) $[P, S]$ is in the Hilbert-Schmidt class, and

(ii) $\quad$ ind $_{P \mathscr{H}} P S P \neq 0$ ?

In [3] and [12], only trivial answers were given; under very restrictive conditions on potentials the index always vanishes.

A possible variant of $(A)$ which seems interesting is the following:

(B) Can we find potentials with scattering operators $S$ satisfying

(i) $[P, S]$ is compact, and

(ii) $\quad$ ind $_{P \mathscr{H}} P S P \neq 0$ ?

The goal of this article is an index formula to the problem (B).

In the massless Dirac case of $1+3$ dimensional space-time, we will consider 
the index corresponding to the chiral charge shift. Under some technical conditions the index of the scattering operator of the Dirac equation will be shown equal to the instanton number,

$$
\operatorname{ind}_{P \mathscr{H}} P S P=-\frac{1}{8 \pi^{2}} \iint_{\mathbb{R}^{4}} \operatorname{Tr} F \wedge F,
$$

where $F$ is the curvature (the energy of the external gauge field) for the connection (gauge potential).

We remark that the integrality of the right-hand side of (1.5) was proved for a fairly large class of potentials by K. Uhlenbeck in [13], but we feel some additional conditions are necessary for the unitarity of $S$ and the compactness of $[P, S]$.

We conclude this introduction by a brief summary of the rest of this article.

In Sect. 2 basic standing assumptions and the main results are presented. Sections 3 and 4 are devoted to the proof of the main theorem. We show that the computation of index of the scattering operator is reduced to that of a pseudodifferential operator in Sect. 3, and we derive an index formula of Toeplitz type operators using a general result of L. Hörmander in Sect. 4. In Sect. 5 we give some remarks.

\section{Statement of Main Results}

We first introduce Dirac operators on $\mathbb{R}^{3}$. We consider both massive and massless cases. For the massless case the basic Hilbert space is $\mathscr{H}=L^{2}\left(\mathbb{R}^{3}\right) \otimes \mathbb{C}^{2} \otimes \mathbb{C}^{N}$, where Pauli matrices (spinors) act on $\mathbb{C}^{2}$ and the $U(N)$ gauge group acts on $\mathbb{C}^{N}$ in the standard way. In the massive case, $\mathscr{H}=L^{2}\left(\mathbb{R}^{3}\right) \otimes \mathbb{C}^{4} \otimes \mathbb{C}^{N}$, and the $4 \times 4$ Dirac matrices act on $\mathbb{C}^{4}$.

The free massless Dirac operator is an essentially selfadjoint operator (on $\left.C_{0}^{\infty}\left(\mathbb{R}^{3}\right) \otimes \mathbb{C}^{2} \otimes \mathbb{C}^{N}\right)$ defined by

$$
H_{0}=\sum_{i=1}^{3} \sigma_{i} p_{i}=\sigma \cdot p
$$

where $p_{k}=-\left(i \partial / \partial x_{k}\right)$ and $\left\{\sigma_{k} ; k=1,2,3\right\}$ are $2 \times 2$ Pauli spin matrices which are selfadjoint and satisfy

$$
\sigma_{k}^{2}=1, \quad \sigma_{k} \sigma_{1}=i \varepsilon_{k l m} \sigma_{m}
$$

( $\varepsilon_{k l m}$ is the totally antisymmetric tensor with $\varepsilon_{123}=1$.)

The massive Dirac operator is defined by

$$
H_{0}=\sum_{k=1}^{3} \alpha_{k} p_{k}+m \beta=\alpha \cdot p+m \beta,
$$

where $m$ is a positive constant corresponding to the mass and $\left\{\alpha_{k} ; k=1,2,3\right\}$ and $\beta$ are $4 \times 4$ Dirac matrices satisfying

$$
\alpha_{k}^{2}=\beta^{2}=1, \quad\left\{\alpha_{k}, \alpha_{1}\right\}=\left\{\alpha_{k}, \beta\right\}=0 \quad(k \neq 1),
$$

where $\{A, B\}=A B+B A$.

Let $A_{k}(t, x)=A_{k}(t)(k=0,1,2,3)$ be skew selfadjoint $N \times N$ matrix valued (time 
dependent) gauge potentials. We assume for simplicity

$$
A_{k}(t) \in C^{\infty}\left(\mathbb{R}^{4}\right) \otimes M_{N} .
$$

( $M_{N}$ is the set of all complex $N \times N$ matrices.)

$A_{k}(t)$ acts as a multiplication operator on $\mathscr{H}$. We use the notation $A_{k}(t)$ or $A_{k}(t, x)$ instead of $1 \otimes A_{k}(t, x)$. The Dirac operator coupled with gauge potentials is defined by

$$
H(t)= \begin{cases}H_{0}+i \sum_{k=1}^{3} \sigma_{k} A_{k}(t)-i A_{0}(t) \quad \text { (massless case) } \\ H_{0}+i \sum_{k=1}^{3} \alpha_{k} A_{k}(t)-i A_{0}(t) \quad \text { (massive case). }\end{cases}
$$

If $A_{k}(t)$ are bounded the domains of $H(t)$ and $H_{0}$ coincide,

$$
D\left(H_{0}\right)=D(H(t)) \text { for arbitrary } t .
$$

The unitary propagator $U(t, s)$ for $H(t)$ is characterized by

(i) $U(t, s)$ is a strongly continuous two parameter unitary,

(ii) $U(t, s) U(s, u)=U(t, u)$,

(iii) $U(t, s) D\left(H_{0}\right)=D\left(H_{0}\right)$,

(iv) $i \frac{\partial}{\partial t} U(t, s) f=H(t) U(t, s) f$,

$$
-i \frac{\partial}{\partial s} U(t, s) f=U(t, s) H(s) f
$$

for $f$ in $D\left(H_{0}\right)$.

The two parameter unitary satisfying $(2.7 a \sim d)$ is unique.

The scattering operator $S$ is defined by

$$
S=\mathrm{st}-\lim _{\substack{t \rightarrow \infty \\ s \rightarrow-\infty}} e^{i t I I_{0}} U(t, s) e^{-i s H_{0}},
$$

provided that the limit exists in the strong operator topology. The projection to the positive spectral subspace of $H_{0}$ is denoted by $P$. By the Fourier transformation $P$ is represented by a multiplication operator $P(\xi)$.

$$
P(\xi)=\left\{\begin{array}{l}
\frac{1}{2}\left\{1+\frac{\sigma \cdot \xi}{|\xi|}\right\} \quad \text { (massless case) } \\
\frac{1}{2}\left\{1+\frac{\alpha \cdot \xi+\beta m}{\left[|\xi|^{2}+m^{2}\right]^{1 / 2}}\right\} \quad \text { (massive case) }
\end{array}\right.
$$

Let $\langle x\rangle=\left[1+\sum_{k=1}^{3} x_{k}^{2}\right]^{1 / 2}$.

Assumption A

(i) $A_{k}(t, x)$ is a smooth bounded function.

(ii) $\left\|A_{k}(t, x)\right\| \leqq C \frac{1}{\langle x\rangle^{\delta_{1}}}$, 
where $C$ and $\delta_{1}$ are some positive constants and $\|\quad\|$ denotes the norm of finite matrices. ( $C$ may depend on $t$.)

(iii) There exist constants $T_{ \pm}$and unitary valued functions $W_{ \pm}(t, x)$ satisfying Assumption $B$ below such that

$$
\left\|A_{k}(t, x)-\left(\partial_{k} W_{+}(t, x)\right) W_{+}(t, x)^{*}\right\| \leqq \frac{1}{\langle x\rangle^{\delta_{2}}} \times G(t) \quad \text { for } \quad t \geqq T_{+},
$$

and

$$
\left\|A_{k}(t, x)-\left(\partial_{k} W_{-}(t, x)\right) W_{-}(t, x)^{*}\right\| \leqq \frac{1}{\langle x\rangle^{\delta_{2}}} \times G(t) \text { for } t \leqq T_{-},
$$

where $\partial_{0}=(\partial / \partial t), \partial_{k}=\left(\partial / \partial x_{k}\right)(k=1,2,3), \delta_{2}$ is a positive constant and $G(t)$ is a positive integrable continuous function on $\mathbb{R}$ satisfying $G(t) \rightarrow 0$ as $|t| \rightarrow \infty$.

(iv) $\delta_{2}$ in (iii) satisfies $\delta_{2}>1$.

(v) Let $A=A_{0}(t, x) d t+\sum_{k=1}^{3} A_{k}(t, x) d x_{k}$ be an 1 form on $\mathbb{R}^{4}$ and $F=d A+A \wedge A$. $F$ is square integrable on $\mathbb{R}^{4}$ and

$$
\|F(t, x)\|<C \frac{1}{\langle x\rangle^{3-\delta_{3}}}
$$

where $C$ is a positive constant independent of $t, \delta_{3}<\min \left(\delta_{2}, 1+\delta\right)$ and $\delta$ is the constant appearing in (2.12).

$$
\lim _{|t| \rightarrow \infty} \int_{\mathbb{R}_{x}^{3}} \operatorname{Tr}(F \Lambda A)=0
$$

\section{Assumption $B$}

Let $W(t, x)$ be a $U(N)$ valued smooth function on $\mathbb{R}^{4}$.

$$
\left\|\partial_{x}^{\alpha}(W(t, x)-1)\right\| \leqq C_{\alpha} \frac{1}{\langle x\rangle^{|\alpha|+\delta}}
$$

where $C_{\alpha}$ and $\delta$ are positive constants independent of $t$ and $\alpha$ is a multi-index,

$$
\alpha=\left(\alpha_{1}, \alpha_{2}, \alpha_{3}\right), \quad \partial_{x}^{\alpha}=\frac{\partial^{\alpha_{1}}}{\partial x_{1}} \frac{\partial^{\alpha_{2}}}{\partial x_{2}} \frac{\partial^{\alpha_{3}}}{\partial x_{3}} .
$$

(ii) The following limit exists uniformly in $v$ in $\mathbb{R}^{3}$.

$$
\lim _{t \rightarrow \pm \infty} W(t, t v)=\tilde{W}_{ \pm}(v)
$$

Theorem 1. Let $A_{k}(t, x)$ satisfy Assumptions $A$ and $B$.

(i) The scattering operator $S$ of $(2.8)$ exists, is unitary and $[P, S]$ is compact.

(ii) PSP restricted to $P \mathscr{H}$ is Fredholm and its index is given by

$$
\text { ind }_{P \mathscr{H}} P S P=\frac{-1}{8 \pi^{2}} \iint_{\mathbb{R}^{4}} \operatorname{Tr} F \wedge F, \quad \text { (massless case), }
$$




$$
\text { ind }_{P \mathscr{H}} P S P=0, \quad \text { (massive case), }
$$

where ind $_{P \mathscr{H}}$ PSP denotes the Fredholm index of the operator PSP restricted to P $\mathscr{H}$. Remark 2.1. The integral in (2.14a) is called the instanton number.

The main part of the proof of Theorem 1 is given in Sects. 3 and 4. In the rest of this section, we give the latter half of the proof assuming the following formula (2.15) whose proof is given in Proposition 3.1,

$$
\operatorname{ind}_{P \mathscr{H}} P S P=-\operatorname{ind}_{P \mathscr{H}} P W_{+}\left(t_{+}\right) P+\operatorname{ind}_{P \mathscr{H}} P W_{-}\left(t_{-}\right) P,
$$

for any $t_{+} \geqq T_{+}$and any $t_{-} \leqq T_{-}$.

In the massive case the right-hand side of (2.15) always vanishes due to Theorem 2 (ii) of Sect. 4 while in the massless case it is equal to

$$
(2.15)=\frac{1}{24 \pi^{2}} \int_{\mathbb{R}^{3}} \operatorname{Tr}\left(\left(d W_{+} W_{+}^{*}\right)^{3}-\left(d W_{-} W_{-}^{*}\right)^{3}\right) .
$$

Note that $d \operatorname{Tr}\left(F \wedge A-\frac{1}{3} A \wedge A \wedge A\right)=\operatorname{Tr} F \wedge F$. (This identity is seen as follows. Using the trace property, $\operatorname{Tr} X Y=(-1)^{\operatorname{deg} X \cdot \operatorname{deg} Y} \operatorname{Tr} Y X$ for matrix valued differential forms. $X, Y$. In particular, $\operatorname{Tr} A^{4}=0$. The rest of the computation is an exercise.) By Green's formula,

$$
\begin{aligned}
\iint_{\mathbb{R}^{4}} \operatorname{Tr}(F \wedge F)= & \lim _{T \rightarrow \infty}\left(\int_{\mathbb{B}_{x}^{3}} \operatorname{Tr}\left(F \wedge A-\frac{1}{3} A \wedge A \wedge A\right)_{t=T}\right. \\
& \left.-\int_{\mathbb{R}_{x}^{3}} \operatorname{Tr}\left(F \wedge A-\frac{1}{3} A \wedge A \wedge A\right)_{t=-T}\right) .
\end{aligned}
$$

By (2.11), (2.12), and

$$
\begin{aligned}
& \operatorname{Tr}\left(A^{3}-\left(d W_{ \pm} W_{ \pm}^{*}\right)^{3}\right)= \operatorname{Tr}\left(\left(A-d W_{ \pm} W_{ \pm}^{*}\right)^{3}+3\left(A-d W_{ \pm} W_{ \pm}^{*}\right)^{2} d W_{ \pm} W_{ \pm}^{*}\right. \\
&\left.+3\left(A-d W_{ \pm} W_{ \pm}^{*}\right)\left(d W_{ \pm} W_{ \pm}^{*}\right)^{2}\right), \\
&\left\|A \wedge A \wedge A-\left(\left(d W_{ \pm}\right) W_{ \pm}^{*}\right)^{3}\right\| \leqq C G(t) \frac{1}{\langle x\rangle^{\rho}}
\end{aligned}
$$

where $\rho=\min \left\{3 \delta_{2}, 2 \delta_{2}+1+\delta, \delta_{2}+2(1+\delta)\right\}$, $\rho>3$, since $\delta_{2}>1$ by our assumptions.

By Assumption $A(\mathrm{v})$ and (vi)

$$
(2.17)=\lim _{T \rightarrow \infty}-\frac{1}{3} \times \int_{\mathbb{R}^{3}} \operatorname{Tr}\left(\left(d W_{+} W_{+}^{*}\right)_{t=T}^{3}-\left(d W_{-} W_{-}^{*}\right)_{t=-T}^{3}\right) .
$$

Thus (2.14a) follows from (2.15).

Example. Let $U(x)$ be a $U(N)$ valued function satisfying assumptions of Theorem 2 of Sect. 4 satisfying

$$
\frac{-1}{24 \pi^{2}} \int_{\mathbb{R}^{3}} \operatorname{Tr}\left(\left(d U U^{*}\right)^{3}=n .\right.
$$

$(U(x)$ satisfying (2.18) exists due to the Remark at the end of Sect. 4.) Set 
$W(t, x)=U(x / t)$, and let $A_{k}(t)$ be potentials satisfying

$$
A_{k}(t, x)=\left\{\begin{array}{cl}
\partial_{k} W(t, x) W(t, x)^{*} & \text { for } t>T_{0}, \\
0 & \text { for } t<0 .
\end{array}\right.
$$

$W(t, x)$ obviously satisfies Assumption $B$ and the potential satisfying (2.18), and Assumption $A$ gives rise to an example of a scattering operator with index $n$ in (2.14a). (Note that $F$ vanishes if $t<T_{0}$ or $T>0$ ).

\section{Proof of Theorem 1}

The aim of this chapter is to give a proof of Theorem 1 (i) and that of the following proposition.

Proposition 3.1. Let $A_{k}(t, x)$ satisfy Assumption $A$ (i) (iii). Then

$$
\operatorname{ind}_{P \mathscr{H}} P S P=-\operatorname{ind}_{P \mathscr{H}} P W_{+}\left(t_{+}\right) P+\operatorname{ind}_{P \mathscr{H}} P W_{-}\left(t_{-}\right) P
$$

for any $t_{+} \geqq T_{+}$and any $t_{-} \leqq T_{-}$.

As explained in Sect. 2, Proposition 3.1 implies Theorem 1 (ii).

We now collect basic properties of Fredholm operators and compact operators which we will use in our proof.

Lemma 3.2. Let $\mathfrak{F}$ be the set of all Fredholm operators equipped with norm topology of bounded operators.

(i) The index map ind: $\mathfrak{F} \longrightarrow Z$ is continuous.

(ii) If $A \in \mathfrak{F}$ and $B$ is compact, then $A+B$ is Fredholm and

$$
\text { ind }(A+B)=\text { ind } A \text {. }
$$

(iii) For $A_{1}, A_{2} \in \mathfrak{F}$, ind $A_{1} A_{2}=$ ind $A_{1}+$ ind $A_{2}$.

\section{Lemma 3.3.}

(i) The set of all compact operators on a Hilbert space is norm closed.

(ii) Let $\Phi(x)$ and $\Psi(\xi)$ be continuous functions of $\mathbb{R}^{n}$ satisfying

$$
|\Phi(x)|<C \frac{1}{\langle x\rangle^{\delta}}, \quad|\Psi(\xi)|<C^{\prime} \frac{1}{\langle\xi\rangle^{\delta^{\prime}}}
$$

for some positive constants $C, C^{\prime}, \delta, \delta^{\prime}$. Let $\Psi\left(D_{x}\right)$ be an operator defined by

$$
\Psi\left(D_{x}\right) u(x)=\iint e^{i \xi x} \Psi(\xi) \tilde{u}(\xi) \frac{d \xi}{(2 \pi)^{n}}
$$

where $u \in L^{2}\left(R^{n}\right), \tilde{u}(\xi)=\iint e^{-i \xi x} u(x) d x$.

Then $\Phi(x) \Psi\left(D_{x}\right)$ is compact.

(iii) A pseudo-differential operator $a\left(x, D_{x}\right)$ with symbol $a(x, \xi)$ in $\mathscr{A}^{-\delta,-\delta^{\prime}}$ is compact if $\delta$ and $\delta^{\prime}$ are strictly positive. (The definition of $\mathscr{A}^{m, m^{\prime}}$ is given in Sect. 4.)

Throughout this chapter we give a proof for the massless case. The massive case may be treated by the same way. 
Lemma 3.4. Let $W(t, x)$ satisfy Assumption $B$. Then,

$$
\text { st- } \lim _{t \rightarrow \pm \infty} e^{i t H_{0}} W(t, x) e^{i t H_{0}}=\tilde{W}_{ \pm}(v)
$$

where $v=H_{0}^{-1} P=P / H_{0}$ and $\tilde{W}_{ \pm}(v)$ is defined in (2.13).

Proof. First note that

$$
e^{i t H_{0}} W(t, x) e^{i t H_{0}}=W(t, t v(t)),
$$

where $v(t)=e^{-i t H_{0}} x / t e^{i t H_{0}}$. For an $\varepsilon>0$ there exists a $T>0$ such that

$$
\left\|W(t, t v(t))-\tilde{W}_{ \pm}(v(t))\right\|\langle\varepsilon \text { for } \pm t\rangle T
$$

due to the uniform convergence of (2.13) and the joint spectral decomposition of commuting operators $v_{k}(t)=e^{-i t H_{0}}\left(x_{k} / t\right) e^{i t H_{0}},(k=1,2,3)$. Thus we have only to show

$$
\text { st- } \lim _{t \rightarrow \pm \infty} \tilde{W}_{ \pm}(v(t))=\tilde{W}_{ \pm}(v) .
$$

By Theorem VIII.20(b) of [9], (3.8) follows from the strong resolvent convergence of $v(t)$ to $v$ which we prove now.

It is easy to check

$$
\left[H_{0}, x_{k}\right]=-i \sigma_{k}, \quad\left[H_{0}, \sigma_{k}\right]=2 H_{0}\left(\sigma_{k}-\frac{p_{k}}{H_{0}}\right) .
$$

By integrating (3.9),

$$
v_{k}(t) u=-\frac{1-e^{-i t H_{0} t}}{2 i H_{0} t}\left(\sigma_{k}-\frac{p_{k}}{H_{0}}\right) u+\frac{p_{k}}{H_{0}} u+\frac{x_{k}}{t} u
$$

for $u \in \mathscr{S}$.

Note that $p_{k} / H_{0}$ and $\left(1-e^{-i 2 H_{0} t} / 2 i H_{0} t\right)$ are bounded operators.

Set

$$
\tilde{u}_{\delta}(\xi)=\mid \begin{array}{cc}
\int e^{-i x \xi} u(x) d x & |\xi| \geqq \delta \\
0 & |\xi|<\delta
\end{array}
$$

and

$$
u_{\delta}(x)=\int e^{i x \xi} \tilde{u}_{\delta}(\xi) d \xi
$$

Then for $\delta$ sufficiently small,

$$
\left\|u_{\delta}-u\right\|_{L^{2}\left(R^{3}\right)}=\left\|\tilde{u}_{\delta}-\tilde{u}\right\|_{L^{2}\left(R^{3}\right)}<\frac{\varepsilon}{6} .
$$

On the other hand

$$
\left\|\frac{1-e^{-i 2 H_{0} t}}{2 i H_{0} t}\left(\sigma_{k}-\frac{p_{k}}{H_{0}}\right) u_{\delta}\right\|_{L^{2}} \leqq \frac{2}{\delta|t|}\left\|u_{\delta}\right\|_{L^{2}},
$$

if $t \neq 0$ because of $\left\|\sigma_{\kappa}\right\|=\left\|\left(p_{k} / H_{0}\right)\right\|=1$ and the support property of $\tilde{u}_{\delta}$. 


\section{Consequently}

$$
\begin{aligned}
& \left\|v(t)_{k} u-\frac{p_{k}}{H_{0}} u\right\|_{L^{2}} \leqq \frac{1}{|t|}\left\|x_{k} u\right\|_{L^{2}}+\left\|\frac{1-e^{-i 2 H_{0} t}}{2 i H_{0}}\left(\sigma_{k}-\frac{p_{k}}{H_{0}}\right)\left(u-u_{\delta}\right)\right\|_{L^{2}} \\
& \quad+\left\|\frac{1-e^{-i 2 H_{0} t}}{2 i H_{0}}\left(\sigma_{\kappa}-\frac{p_{k}}{H_{0}}\right) u_{\delta}\right\|_{L^{2}} \\
& \leqq \frac{1}{|t|}\left\|x_{k} u\right\|_{L^{2}}+2\left\|u_{\delta}-u\right\|_{L^{2}}+\frac{2}{\delta|t|}\left\|u_{\delta}\right\|_{L^{2}}<\varepsilon,
\end{aligned}
$$

provided that $|t|>T, T=\max \left\{(3 / \varepsilon)\left\|x_{k} u\right\|_{L^{2}},(6 / \delta \varepsilon)\left\|u_{\delta}\right\|_{L^{2}}\right\}$.

This proves

$$
\lim _{|t| \rightarrow \infty} v(t) u=v u \text { for } \quad u \in \mathscr{S} .
$$

Next

$$
\left\|\left(\frac{1}{v(t)_{k}+i}-\frac{1}{v_{k}+i}\right) u\right\|_{L^{2}}=\|\left(\frac{1}{v_{k}(t)+i}\left(v_{k}-v_{k}(t)\right) \frac{1}{v_{k}+i} u\left\|_{L^{2}} \leqq\right\|\left(v_{k}-v_{k}(t)\right) \frac{1}{v_{k}+i} u \|_{L^{2}} .\right.
$$

Since $\mathscr{S}$ is dense in $L^{2}\left(R^{3}\right)$, (3.13) and (3.14) imply the strong resolvent convergence of $v_{k}(t)$ to $v_{k}$. This completes the proof of Lemma 3.4.

Lemma 3.5. Let $A_{k}(t, x)$ satisfy Assumption $A$ (i) (iii). The scattering operator exists, is unitary and is represented by the formula:

$$
S=e^{i T_{+} H_{0}} \tilde{W}_{+}(v) W_{+}\left(T_{+}, x\right)^{*} V_{+} U\left(T_{+}, T_{-}\right) V_{-} W_{-}\left(T_{-}, x\right) \tilde{W}_{-}(v)^{*} e^{i T_{-} H_{0}},
$$

where $v=\left(p / H_{0}\right)$, and

$$
\begin{aligned}
V_{+}= & 1+\sum_{n=1}^{\infty} \int_{T_{+} \leqq s_{n} \leqq s_{n-1} \leqq \cdots \leqq s_{1}<\infty} W\left(T_{+}, x\right) e^{-i\left(T_{+}-s_{1}\right) H_{0}} \\
& \cdot W\left(s_{1}, x\right)^{*} B_{+}\left(s_{1}, x\right) W\left(s_{1}, x\right) e^{-i\left(s_{1}-s_{2}\right) H_{0}} W\left(s_{2}, x\right)^{*} \cdots B_{+}\left(s_{n}, x\right) W\left(s_{n}, x\right) \\
& \cdot e^{-i\left(s_{n}-T_{+}\right) H_{0}} W\left(T_{+}, x\right) d s_{1} \cdots d s_{n} \\
V_{-}= & 1+\sum_{n=1}^{\infty}(-1)^{n} \int_{-\infty \leqq s_{1} \leqq s_{2} \leqq \cdots \leqq s_{n} \leqq T_{-}} W\left(T_{-}, x\right)^{*} e^{-i\left(T_{-}-s_{n}\right) H_{0}} \\
& \cdot W\left(s_{n}, x\right) B_{-}\left(s_{n}, x\right) \cdots B_{-}\left(s_{1}, x\right) W\left(s_{1}, x\right) e^{-i\left(T_{-}-s_{1}\right) H_{0}} \\
& \cdot W\left(T_{-}, x\right)^{*} d s_{1} d s_{2} \cdots d s_{n}, \\
B_{ \pm}(s, x)= & \sum_{k=1}^{3} \sigma_{k}\left(A_{k}(s, x)-\partial_{k} W_{ \pm}(s, x) W_{ \pm}(s, x)^{*}\right)+\left(A_{0}(s, x)-\partial_{0} W(s, x) W(s, x)^{*}\right) .
\end{aligned}
$$

Proof. First we remark that (3.16) is convergent. In fact, by (iii) of Assumption $A$ 
the operator norm of $n$-th term in (3.16a) is bounded above by

$$
C^{n} \int_{T_{+} \leqq s_{n} \leqq \cdots \leqq s_{2} \leqq s_{1}<\infty} G\left(s_{1}\right) G\left(s_{2}\right) \cdots G\left(s_{n}\right) d s_{1} d s_{2} \cdots d s_{n}=\frac{1}{n !}\left(C \int_{T_{+}}^{\infty} G(s) d s\right)^{n} .
$$

(The integral may be carried out inductively.)

These same estimate works for (3.16b) and as a consequence,

$$
\left\|V_{ \pm}\right\| \leqq \exp \left(C\|G\|_{L^{1}\left(\mathbb{R}^{1}\right)}\right) \text {. }
$$

Next by the identity,

$$
e^{i t H_{0}} U(t,-s) e^{i s H_{0}}=\left(e^{i t H_{0}} U\left(t, T_{+}\right)\right) U\left(T_{+}, T_{-}\right)\left(U\left(T_{-},-s\right) e^{i s H_{0}}\right) .
$$

We have only to show that existence and the unitarity of

$$
\lim _{t \rightarrow \infty} e^{i t H_{0}} U\left(t, T_{+}\right) \text {and } \lim _{t \rightarrow \infty} U\left(T_{-},-t\right) e^{i t H_{0}} .
$$

Let $A_{k}^{(1)}$ and $A_{k}^{(2)}$ be gauge potentials, $U^{1}(t, s) U^{2}(t, s)$ be corresponding propagators for Dirac equations.

We use the Dyson expansion for $U^{1}(t, s)^{*} U^{2}(t, s)$. More precisely we consider $U^{1}(t, s)^{*} U^{2}(t, s)$ as the unique solution of the following equation with the initial condition $U^{1}(s, s)^{*} U^{2}(s, s)=1$,

$$
\frac{d}{d t}\left(U^{1}(t, s)^{*} U^{2}(t, s)\right)=U^{1}(t, s)^{*} X(t) U^{1}(t, s)\left(U^{1}(t, s)^{*} U^{2}(t, s)\right),
$$

where

$$
X(t)=\left\{\sigma \cdot\left(\mathbb{A}^{(2)}(t)-\mathbb{A}^{(1)}(t)\right)+A_{0}^{(2)}(t)-A_{0}^{(1)}(t)\right\} .
$$

The integration of (3.18a) leads to

$$
\begin{gathered}
U^{1}(t, s)^{*} U^{2}(t, s)=1+\sum_{n=1}^{\infty} \int_{\substack{s \leqq t_{n} \leqq t_{n-1} \leqq \cdots t_{1} \leqq t\\
}} U^{1}\left(t_{1}, s\right)^{*} X\left(t_{1}\right) U^{1}\left(t_{1}, t_{2}\right) X\left(t_{2}\right) \cdots \\
\cdot X\left(t_{n}\right) U^{1}\left(t_{n}, s\right) d t_{1} d t_{2} \cdots d t_{n} .
\end{gathered}
$$

We set $A_{k}^{(1)}=\partial_{k} W_{+} W_{+}^{*}, A_{k}^{(2)}=A_{k}$. Obviously $U^{(2)}(t, s)=U(t, s)$, and it is easy to check

$$
U^{1}(t, s)=W_{+}(t, x) e^{-i(t-s) H_{0}} W_{+}(s, x)^{*} .
$$

Then

$$
e^{i t H_{0}} U\left(t, T_{+}\right)=e^{i t H_{0}} W_{+}(t) e^{-i t H_{0}} e^{\imath T_{+} H_{0}} W_{+}\left(T_{+}\right)^{*}\left(U^{1}\left(t, T_{+}\right)^{*} U^{2}(t, T)\right) .
$$

The limit of $e^{i t H_{0}} W_{+}(t) e^{-i t H_{0}}$ was computed in the previous lemma. By (3.19), $\lim _{t \rightarrow \infty} U^{1}\left(t, T_{+}\right)^{*} U^{2}\left(t, T_{+}\right)=V_{+}$. Thus

$$
\lim _{t \rightarrow \infty} e^{i t H_{0}} U(t, T)=e^{i *{ }_{r} H_{0}} \tilde{W}_{+}(v) W\left(T_{+}, x\right)^{*} V_{+} .
$$

(Note that $\left[v, H_{0}\right]=0$ implies $\left.\left[e^{i t H_{0}}, \tilde{W}_{+}(v)\right]=0.\right) V_{+}$is an isometry as it is a limit of unitaries. If we consider $V_{+}^{*}$, it is also an isometry. This tells us that $V_{+}$is unitary.

The same argument works for the limit of $U\left(T_{-},-s\right) e^{i s H_{0}}$. 
Lemma 3.6. $\left[W_{ \pm}(t, x), P\right]$ is compact.

Proof. Let $\varphi \in C^{\infty}\left(\mathbb{R}^{3}\right)$ satisfying $\varphi(\xi)=1$ if $|\xi|>1,=0$ if $|\xi| \leqq \frac{1}{2}$. Then

$$
\left[W_{ \pm}(t, x), P\right]=\left[\left(W_{ \pm}(t, x)-1\right), \varphi\left(D_{x}\right) P\right]+\left[\left(W_{ \pm}(t, x)-1\right),\left(1-\varphi\left(D_{x}\right) P\right] .\right.
$$

The second term is compact because $\left(W_{ \pm}(t, x)-\mathbb{1}\right)\left(\mathbb{1}-\varphi\left(D_{x}\right)\right)$ and $\left(\mathbb{1}-\varphi\left(D_{x}\right)\right)$ $\left(W_{ \pm}(t, x)-1\right)$ are compact due to Lemma 3.3 (ii).

Next note that $\varphi\left(D_{x}\right) P, W_{ \pm}(t, x)-1$ are pseudo-differential operators with symbol in $A^{0,0}$. (See Sect. 4.) The commutator of these operators is also a pseudo-differential operator with symbol in $A^{-1,-1}$. (The symbols of the above operators commute as matrices and the term in $A^{0,0}$ of the asymptotic expansion vanishes.)

Combined with Lemma 3.3 (iii) this proves the compactness of the first term of (3.21).

(q.e.d.)

Lemma 3.7. Let $A_{k}(t, x)$ satisfy Assumption $A$ (i) $\sim($ iii). Then $[P, U(t, s)]$ is compact. Proof. Recall that $e^{i t H_{0}} U(t, s) e^{-i s H_{0}}$ is given by the norm convergent Dyson expansion:

$$
\begin{aligned}
e^{i t H_{0}} U(t, s) e^{-i s H_{0}}= & \sum_{n=0}^{\infty} \int_{s \leqq t_{n} \leqq t_{n}-\cdots \leqq t_{1} \leqq t} e^{i\left(t_{1}-s\right) H_{0}} X\left(t_{1}\right) \\
& \cdot e^{-i\left(t_{1}-t_{2}\right) H_{0}} X\left(t_{2}\right) \cdots X\left(t_{n}\right) e^{-i t_{n} H_{0}} d t_{1} d t_{2} \cdots d t_{n} .
\end{aligned}
$$

If $X(t, x)$ defined in (3.18b) is compactly supported in $x$, the commutator $[P, X(t)]$ is compact by the same proof as in Lemma 3.6. The norm convergence of (3.22) suggests the claim. The case that $X(t, x)$ is not compactly supported in $x$ may be proved as follows.

Let $\psi$ in $C^{\infty}\left(\mathbb{R}^{3}\right)$ satisfying

$$
\psi(x)=\left\{\begin{array}{l}
0|x| \geqq 2 \\
1|x| \leqq 1
\end{array}, \quad 0 \leqq \psi \leqq 1,\right.
$$

Let $U^{R}(t, s)$ be the propagator with $A(t)$ replaced by $A(t) \psi(x / R)$. As $A(t) \psi(x / R)$ is compactly supported in $x,\left[P, U^{R}(t, s)\right]$ is compact, as we have already seen. By Assumption $A$ (ii),

$$
\left\|X(t, x)-X_{R}(t, x)\right\| \leqq C \frac{1}{R^{\delta}},
$$

where $C$ is a constant independent of $t$ and $x$.

By (3.22) and (3.24)

$$
\text { norm- } \lim _{R \rightarrow \infty}\left[P, U^{R}(t, s)\right]=[P, U(t, s)] .
$$

As the left-hand side of (3.25) is compact, the proof is completed.

Lemma 3.8. $\left[P, V_{ \pm}\right]$is compact.

Proof. By (3.16a),

$$
V_{+}=W\left(T_{+}, x\right) e^{-i T_{+} H_{0}} \tilde{V}_{+} e^{i T_{+} H_{0}} W\left(T_{+}, X\right)^{*},
$$




$$
\tilde{V}_{+}=\sum_{n=0}^{\infty} \int_{T_{+} \leqq s_{n} \leqq s_{1}<\infty} e^{i s_{1} H_{0}} \tilde{B}\left(s_{1}, x\right) e^{-i s_{1} H_{0} \cdots} \tilde{B}\left(s_{n}, x\right) e^{-i s_{n} H_{0}} d s_{1} d s_{2} \cdots d s_{n},
$$

where $\tilde{B}(s, x)=W(s, x)^{*} B(s, x) W(s, x)$.

It suffices to show that $\left[P, \widetilde{V}_{+}\right]$is compact, since $\left[P, W\left(T_{ \pm}\right)\right]$is compact by Lemma 3.6.

Consider the operator

$$
\left[P, e^{i s H_{0}} \widetilde{B}(s, x) e^{-i s H_{0}} \ldots \widetilde{B}\left(s_{n}, x\right) e^{-i s_{n} H_{0}}\right] .
$$

$[P, \widetilde{B}(s, x)]$ is compact by the same reasoning as in the proof of Lemma 3.7. By the identity $[A, B C]=[A, B] C+B[A, C],(3.27)$ is compact. As (3.26) is norm convergent by Assumption $A$ (iii) $\left[P, \widetilde{V}_{+}\right]$is compact. The same argument leads to the compactness of $\left[P, \tilde{V}_{-}\right]$.

Lemmas 3.4. 3.8 imply Theorem 1 (i). Next we show Proposition 3.1.

Lemma 3.9. Suppose that $U_{1}, U_{2}$ are unitary and $\left[P, U_{i}\right](i=1,2)$ are compact. Then $P U_{k} P(k=1,2), P U_{1} U_{2} P$ on $P \mathscr{H}$ are Fredholm and

$$
\operatorname{ind}_{P \mathscr{H}} P U_{1} U_{2} P=\operatorname{ind}_{P \mathscr{H}} P U_{1} P+\operatorname{ind}_{P \mathscr{H}} P U_{2} P \text {. }
$$

Proof. To see $P U_{j} P$ is Fredholm, it suffices to show that it is invertible modulo compact operator. In fact $P U_{j}^{*} P$ is the inverse of $P U_{j} P$ in the following sense:

$$
\begin{aligned}
& P U_{j}^{*} P U_{j} P=P-P\left[P, U_{j}^{*}\right]\left[P, U_{j}\right] P, \\
& P U_{j} P U_{j}^{*} P=P-P\left[P, U_{j}\right]\left[P, U_{j}^{*}\right] P .
\end{aligned}
$$

Next note that $P U_{1} U_{2} P-P U_{1} P U_{2} P=P\left[P, U_{1}\right] U_{2} P$ is compact. Equation (3.28) is valid due to Lemma 3.2 (ii) and (iii).

Proof of Proposition 3.1. By Lemmas 3.5 and 3.9 it suffices to show

$$
\text { ind }_{P \mathscr{H}} P U(t, s) P=\operatorname{ind}_{P \mathscr{H}} P V_{ \pm} P=0 \text {. }
$$

As the Dyson expansion for $e^{i t H_{0}} U(t, s) e^{-i s H_{0}}$ is norm convergent we have the estimate

$$
\left\|e^{i t H_{0}} U(t, s) e^{-i s H_{0}}-\mathbb{1}\right\| \leqq C\left(e^{K|t-s|}-1\right) .
$$

By $\left[P, e^{i t H_{0}}\right]=0$, and Lemma 3.2 (i),

$$
\begin{aligned}
& \operatorname{ind}_{P \mathscr{H}} P U(t, s) P=\operatorname{ind}_{P \mathscr{H}}\left(P e^{i t H_{0}} U(t, s) e^{-i s H_{0}} P\right) \\
& =\lim _{t \rightarrow s} \operatorname{ind}_{P \mathscr{H}}\left(P e^{i t H_{0}} U(t, s) e^{-i s H_{0}} P\right)=0 .
\end{aligned}
$$

Next let $V_{ \pm}^{\lambda}$ be defined by (3.26) replacing $B(s, x)$ with $\lambda B(s, x)$. It is easy to show that $V_{ \pm}^{\lambda}$ is norm continuous in $\lambda$. Thus,

$$
\operatorname{ind}_{P \mathscr{H}} P V_{ \pm} P=\operatorname{limind}_{\lambda \rightarrow 0} P V_{ \pm}^{\lambda} P=0 \text {. }
$$

This completes the proof of Proposition 3.1.

Remark. Hilbert norm estimates of $[P, S]$ are given in $[8,11,12]$ under more restrictive situations. 


\section{An Index Formula for Gauge Groups}

Theorem 2. Let $U(x)$ be a $U(N)$ valued smooth function on $\mathbb{R}^{3}$ acting as a multiplication operator on $\mathscr{H}$. Suppose that there exist positive constants $\delta$ and $C_{\alpha}$ such that

$$
\left\|\partial_{x}^{\alpha}(U(x)-1)\right\| \leqq C_{\alpha} \frac{1}{\langle x\rangle^{\delta+|\alpha|}}
$$

for an arbitrary multi-index $\alpha=\left(\alpha_{1}, \alpha_{2}, \alpha_{3}\right)$. Then $P U P$ restricted to $P \mathscr{H}$ is a Fredholm operator and,

(i) $\operatorname{ind}_{P \mathscr{H}} P U P=\frac{-1}{24 \pi^{2}} \iint_{\mathbb{R}^{3}} \operatorname{Tr}\left(\left(d U \cdot U^{*}\right)^{3}\right)$ for the massless case.

(ii) ind $_{P \mathscr{H}} P U P=0$ for the massive case.

We remark that the integral of $(4.2 \mathrm{a})$ gives rise to an element of $\pi_{3}(U(N))=\mathbb{Z}$, where $\mathbb{R}^{3} \cup\{\infty\}$ is identified with $S^{3}$. On the odd dimensional compact $\operatorname{spin}^{c}$ manifold, the formula corresponding to (4.2a) is a special case of Atiyah-Singer's index theorem. See [2]. We derive (4.1) from a result of L. Hörmander in [5].

First we introduce a symbol class of pseudo-differential operators. For real numbers $m$ and $m^{\prime}$ we define $\mathscr{A}^{m, m^{\prime}}$ by

$$
\mathscr{A}^{m, m^{\prime}}=\left\{a(x, \xi) \quad \text { in } \quad C^{\infty}\left(\mathbb{R}^{2 n}\right):\left|\partial_{x}^{\alpha} \partial_{\xi}^{\beta} a(x, \xi)\right| \leqq C_{\alpha, \beta}\langle x\rangle^{m-|\alpha|}\langle\xi\rangle^{m^{\prime}-|\beta|}\right\} .
$$

For $a(x, \xi)$ in $\mathscr{A}^{m, m^{\prime}}$, the pseudo-differential operator $a\left(x, D_{x}\right)$ is defined by

$$
a\left(x, D_{x}\right) u(x)=\text { Os- } \iint e^{i \xi \cdot x} a(x, \xi) \tilde{u}(\xi) \frac{d \xi}{(2 \pi)^{n}}
$$

for $u$ in $\mathscr{S}\left(\mathbb{R}^{n}\right)$ where Os- $\int$ denotes the oscillatory integral, and $\tilde{u}(\xi)=\int e^{-i \xi \cdot x} u(x) d x$. $a\left(x, D_{x}\right)$ extends to a bounded operator on $L^{2}\left(R^{n}\right)$ if both $m$ and $m^{\prime}$ are non-positive.

The following formula is fundamental.

Product of Pseudo-differential operators. Let $a_{1}(x, \xi)$ be in $\mathscr{A}^{m_{i}},,^{n_{z}}(i=1,2)$. Then there exists a symbol $b(x, \xi)$ in $\mathscr{A}^{m_{1}+m_{2}, n_{1}+n_{2}}$ such that

$$
a_{1}\left(x, D_{x}\right) a_{2}\left(x, D_{x}\right)=b\left(x, D_{x}\right)
$$

and

$$
r_{N}(x, \xi) \equiv b(x, \xi)-\sum_{|\alpha|<N} \frac{(-i)^{|\alpha|}}{\alpha !} \partial_{\xi}^{\alpha} a_{1}(x, \xi) \cdot \partial_{x}^{\alpha} a_{2}(x, \xi) \in \mathscr{A}^{m_{1}+m_{2}-N, n_{1}+n_{2}-N} .
$$

For the proof, see [5] or modify the proof for $S_{\rho, \delta}^{m}$ in [6].

L. Hörmander has computed the index of elliptic pseudo-differential operators on $\mathbb{R}^{n}$ in [5].

Theorem 3. If $a(x, \xi)$ in $\mathscr{A}^{0,0}$ takes values in $k \times k$ matrices such that $a(x, \xi)^{-1}$ exists, is bounded outside an open ball $B$ in $\mathbb{R}^{2 n}$, then $a\left(x, D_{x}\right)$ is a Fredholm operator in $L^{2}\left(\mathbb{R}^{n}\right) \otimes \mathbb{C}^{k}$ with index

$$
\text { ind } a\left(x, D_{x}\right)=(-1)\left(\frac{-1}{2 \pi i}\right)^{n} \frac{(n-1) !}{(2 n-1) !} \iint_{\partial B} \operatorname{Tr}\left(\left(a^{-1} d a\right)^{2 n-1}\right)
$$


if $\mathbb{R}^{2 n}$ is oriented by $d x_{1} \wedge d \xi_{1} \wedge d x_{2} \wedge d \xi_{2} \cdots \wedge d \xi_{n}>0$.

(See Theorem 19.3.1 and its remark of [5] and also [4].)

We study first massless case in Lemmas 4.1 and 4.2.

Lemma 4.1. Let $\varphi$ be a smooth function on $\mathbb{R}$ satisfying

$0 \leqq \varphi(x) \leqq 1, \quad \varphi(x)=0 \quad$ (if $\left.\quad x \leqq \frac{1}{2}\right),=1 \quad$ (if $\quad x \geqq 1$ ). Set $P_{\varphi}(\xi)=P(\xi) \varphi(|\xi|)$.

Then $a(x, \xi) \equiv \mathbb{1}+(U(x)-1) P_{\varphi}(\xi)$ satisfies assumptions of Theorem 3 and

$$
\operatorname{ind}_{P \mathscr{H}} P U P=\operatorname{ind}_{\mathscr{H}}\left\{\mathbb{1}+(U-1) P_{\varphi}\left(x, D_{x}\right)\right\} \text {. }
$$

Proof. Clearly ind $\operatorname{ifH}_{\mathscr{H}} P U P=\operatorname{ind}_{\mathscr{H}}[(\mathbb{1}-P)+P U P]$. Hence (4.6) is proved if $\{(\mathbb{1}-P)+$ $P U P\}-\left\{1+(U-\mathbb{1}) P_{\varphi}\right\}$ is compact. In fact,

$$
\{(\mathbb{1}-P)+P U P\}-\left\{\mathbb{1}+(U-\mathbb{1}) P_{\varphi}\right\}=(U-\mathbb{1})(\mathbb{1}-\varphi)\left(x, D_{x}\right) P+[P, U] P .
$$

$(U-1)(1-\varphi)\left(x, D_{x}\right)$ and $[P, U]$ are compact due to Lemma 3.3. (iii) and the proof of Lemma 3.6. Thus (4.8) is verified. $a(x, \xi)$ is obviously in $\mathscr{A}^{0,0}$. Next we check that $a(x, \xi)$ has a bounded inverse outside a compact set of $\mathbb{R}^{2 n}$. To see this, let $b(x, \xi)$ be a symbol in $\mathscr{A}^{0,0}$ determined by

$$
b(x, \xi)=1+\left(U(x)^{*}-1\right) P_{\varphi}(\xi) .
$$

If $|\xi| \geqq 1$

$$
a(x, \xi) b(x, \xi)=b(x, \xi) a(x, \xi)=1 .
$$

Let $R_{0}$ be a constant sufficiently large that $\|U(x)-1\|<1$ if $|x|<R_{0}$. Then by the Neumann expansion,

$$
a(x, \xi)^{-1}=1+\sum_{n=1}^{\infty}(-1)^{n}(U(x)-1)^{n} P_{\varphi} \varphi(\xi)^{n-1}
$$

for $|x|>R_{0}$.

Thus $a(x, \xi)$ has the bounded inverse on $\{|\xi| \geqq 1\} \cup\left\{|x|>R_{0}\right\}$.

Lemma 4.2. Let $a(x, \xi)$ and $b(x, \xi)$ be defined as above. Then

$$
\text { ind } a\left(x, D_{x}\right)=\frac{(-2 i)}{(2 \pi)^{3} \cdot 5 !} \int_{\mathbb{R}^{3} \times\{\xi:|\xi|<1\}} \operatorname{Tr}\left((d a d b)^{3}\right) \text {, }
$$

where the orientation is same as in Theorem 3.

Proof. By Theorem 3

$$
\text { ind } a\left(x, D_{x}\right)=\frac{(-2)}{(-2 \pi i)^{3} \cdot 5 !} \iint_{\partial B_{R}} \operatorname{Tr}\left(\left(a^{-1} d a\right)^{5}\right) \text {, }
$$

where $B_{R}$ is the ball of radius $R$ centered at origin and $R>R_{0}+1$. As $a^{-1} d a a^{-1}=$ $-d\left(a^{-1}\right)$

$$
\iint_{\partial B_{R}} \operatorname{Tr}\left(\left(a^{-1} d a\right)^{5}\right)=\iint_{\partial B_{R}} \operatorname{Tr}\left(a^{-1}\left(d a\left(d a^{-1}\right)\right)^{2} d a\right) .
$$


We first prove

$$
\left|\iint_{\partial B_{R}}\left\{\operatorname{Tr}\left(a^{-1}\left(d a d a^{-1}\right)^{2} d a\right)-\operatorname{Tr}\left(b(d a d b)^{2} d a\right)\right\}\right|<C_{1} \frac{1}{R^{\delta}} .
$$

As $b(x, \xi)=a^{-1}(x, \xi)$ if $|\xi| \geqq 1$ by (4.11), we estimate the contribution of the integral from $|\xi|<1,|x|^{2}=R^{2}-|\xi|_{0}^{2}>R$. As $a(x, \xi)$ is in $\mathscr{A}^{0,0}$,

$$
\left\|\partial_{x_{k}} a(x, \xi)\right\| \leqq C_{2} \frac{1}{\langle x\rangle^{1+\delta}}, \quad\left\|\partial_{x_{k}} b(x, \xi)\right\| \leqq C_{3} \frac{1}{\langle x\rangle^{1+\delta}} .
$$

On the other hand

$$
U(x)^{*}=\sum_{n=0}^{\infty}(-1)^{n}(U(x)-\mathbb{1})^{n}
$$

if $|x|>R_{0}$ due to $U(x)^{*}=[\mathbb{1}+(U(x)-1)]^{-1}$.

Thus by (4.12) and (4.18) the following estimates hold if $|x|>R_{0}$.

$$
\begin{aligned}
a(x, \xi)^{-1}-b(x, \xi) & =\sum_{n=2}^{\infty}(-1)^{n}(U(x)-1)^{n} P_{\varphi}\left\{\varphi(|\xi|)^{n-1}-1\right\} \\
\left\|\partial_{x_{k}}\left(a(x, \xi)^{-1}-b(x, \xi)\right)\right\| & \leqq \sum_{n=2}^{\infty} n \times\left\|\partial_{x_{k}} U(x)\right\|\|U(x)-1\|^{n-1}|\varphi(|\xi|)-1| \\
& \leqq C_{4} \frac{|\varphi(|\xi|)-1|}{\langle x\rangle^{1+\delta}} \\
\| \partial_{\xi_{k}}\left(a(x, \xi)^{-1}-b(x, \xi) \|\right. & \leqq C_{5} \sum_{n=2}^{\infty} n\|U(x)-1\|^{n} \Phi(\xi) \leqq C_{6} \frac{1}{\langle x\rangle^{\delta}} \Phi(\xi)
\end{aligned}
$$

where $\Phi(\xi)$ is a smooth function supported in $\{|\xi| \leqq 1\}$.

$$
\left\|a(x, \xi)^{-1}-b(x, \xi)\right\| \leqq C_{7} \frac{1}{\langle x\rangle^{\delta}}|\varphi(|\xi|)-1|
$$

Consider the integral of the type

$$
\int_{\partial B_{R} \cap\{|\xi| \leqq 1\}} \operatorname{Tr}\left(c(d a d c)^{2} d a\right) \quad c=a^{-1} \text { or } b .
$$

The integrand is differentiated in $x$ directions more than twice. Taking into account of (4.16) and (4.19b) the above integral is uniformly bounded in $R$. This estimate and (4.18) imply (4.16) due to the following identity,

$$
\begin{aligned}
& \left\{a^{-1}\left(d a d a^{-1}\right)^{2} d a\right\}-\left\{b(d a d b)^{2} d a\right\} \\
& \quad=\left(a^{-1}-b\right)\left(d a d a^{-1}\right)^{2} d a+b d a d\left(a^{-1}-b\right) d a d a^{-1} d a+b d a d b d a d\left(a^{-1}-b\right) d a .
\end{aligned}
$$

Next note that $d\left[\operatorname{Tr}\left(b(d a d b)^{2} d a\right)\right]=-\operatorname{Tr}(d a d b)^{3}$. Then

$$
\iint_{\partial B_{R}} \operatorname{Tr}\left(b(d a d b)^{2} d a\right)=-\iint_{B_{R}} \operatorname{Tr}(d a d b)^{3} .
$$

Using $d b=-a^{-1} d a \cdot a^{-1}(|\xi| \geqq 1)$, anticommutativity of one forms and trace 
property lead to $\operatorname{Tr}\left((d a d b)^{3}\right)=-\operatorname{Tr}\left(d a \cdot a^{-1}\right)^{6}=0$. Thus

$$
(4.20)=-\int_{|\xi| \leqq 1,|x|^{2}+|\xi|^{2} \leqq R^{2}} \operatorname{Tr}(d a d b)^{3} .
$$

By (4.14) (4.16) and (4.20),

$$
\mid \text { ind } a\left(x, D_{x}\right)-\frac{(-2 i)}{(2 \pi)^{3} \cdot 5 !} \iint_{|\xi| \leqq 1,|x|^{2}+|\xi|^{2} \leqq R^{2}} \operatorname{Tr}(d a d b)^{3} \mid \leqq C \frac{1}{R^{\delta}} .
$$

Letting $R$ tend to infinity, we obtain (4.13).

Proof of Theorem 2. The Fredholm property is due to unitarity of $U(x)$ and compactness of $[P, U(x)]$ (see Lemma 3.9). We prove (4.2a) first. By definition,

$$
d a=(U-1) d P_{\varphi}+P_{\varphi} d U, \quad d b=\left(U^{*}-1\right) d P_{\varphi}+P_{\varphi} d U^{*}
$$

Thus

$$
(d a d b)=\left[\left(\mathbb{1}-U^{*}\right) d P_{\varphi}+P_{\varphi} d U \cdot U^{*}\right]\left[(\mathbb{1}-U) d P_{\varphi}-P_{\varphi} d U \cdot U^{*}\right] .
$$

In the integration of $\xi$ coordinate in (4.13) only the following terms occur,

$$
\begin{aligned}
\iint_{|\xi| \leqq 1} \operatorname{Tr}\left(\left(d P_{\varphi}\right)^{2} P_{\varphi} d P_{\varphi} P_{\varphi} \cdot \varphi\right)= & \iint \varphi^{5} d \varphi \operatorname{Tr}\left((d P)^{2} P\right)=\frac{\pi i}{3}, \\
\iint_{|\xi| \leqq 1} \operatorname{Tr}\left(\left(d P_{\varphi}\right)^{3} P_{\varphi} \cdot \varphi^{2}\right)= & i \iint \varphi^{5} d \varphi \operatorname{Tr}\left((d P)^{2} P\right) \\
& +\iint \varphi^{6} \operatorname{Tr}\left((d P)^{3} P\right) \\
= & \frac{2 \pi i}{3}, \\
\iint_{|\xi| \leqq 1} \operatorname{Tr}\left(\left(d P_{\varphi} P_{\varphi}\right)^{3}\right)= & 0 .
\end{aligned}
$$

(The integrals of (4.23) may be carried out by the polar coordinate.)

By (4.14) and (4.22),

$$
\text { ind } a\left(x, D_{x}\right)=\frac{(-2 i)}{(2 \pi)^{3} \cdot 5 !} \int_{\{|\xi| \leqq 1\} \times R^{3}} \operatorname{Tr}\left[\left(A^{*} d P_{\varphi}+P_{\varphi} B\right)\left(A d P_{\varphi}-P_{\varphi} B\right)\right]^{3} \text {, }
$$

where $A=1-U, B=d U \cdot U^{*}$.

The integrand of (4.24) consists of ${ }_{6} C_{3}(=20)$ terms. After using trace property and anticommutativity of 1 forms, we obtain

$$
\operatorname{Tr}\left\{3\left(d P_{\varphi}\right)^{3} P_{\varphi} \varphi^{2} \cdot A A^{*}\left(A+A^{*}\right) B^{3}+6\left(d P_{\varphi}\right)^{2} P_{\varphi} d P_{\varphi} P_{\varphi} \varphi \cdot A A^{*} B\left(A+A^{*}\right) B^{2}\right\}
$$

Recall that $A A^{*}=A^{*} A=A+A^{*}=21-U-U^{*}$.

Next we give several formulae of differential forms,

$$
\int \operatorname{Tr} U B^{3}=\int \operatorname{Tr}\left(d U \cdot U^{*} d U \cdot U^{*} d U\right)=-\int \operatorname{Tr}\left(d U d U^{*} d U\right)=-\int d\left(\operatorname{Tr}\left(U d U^{*} d U\right)\right)=0 .
$$


Similarly

$$
\int \operatorname{Tr} U^{*} B^{3}=0
$$

We now show

$$
\int \operatorname{Tr}\left[U B U^{*} B^{2}+U^{*} B U B^{2}\right]=0 .
$$

Equation (4.27) is a consequence of the following two identities:

$$
\begin{aligned}
& \int \operatorname{Tr}\left(d U d U U^{*} d U^{*}-U d U d U^{*} d U^{*}\right)=\int \operatorname{Tr}\left(d\left(U d U U^{*}\right) d U^{*}\right) \\
&=\int \mathrm{d} \operatorname{Tr}\left(U d U U^{*} d U^{*}\right)=0, \\
& \int \operatorname{Tr} U B U^{*} B^{2}=\int \operatorname{Tr}\left(d U U^{*}\right)\left(U^{*} d U U^{*}\right) d U=-\int \operatorname{Tr}\left(d U d U U^{*} d U^{*}\right) .
\end{aligned}
$$

Finally

$\int \operatorname{Tr}\left(U B U B^{2}+U^{2} B^{3}\right)=-\int \mathrm{d} \operatorname{Tr}\left(U^{2}\left(d U d U^{*}+d U^{*} d U\right)\right)+\int \mathrm{d} \operatorname{Tr}\left(U^{2} d U^{*} d U\right)=0$.

By $(4.25) \sim(4.28)$,

$$
\int \operatorname{Tr}(d a d b)^{3}=(-2 \pi i) \times 10 \times \int B^{3} .
$$

This proves $(4.2 \mathrm{a})$.

Next we prove $(4.2 b)$.

Recall that $P$ is given by the formula (2.9b) and (3.5). Let $P_{0}$ be a projection given by the symbol $P(\xi)$ with $m=0$. Then $\{1+(U-1) P\}-\left\{1+(U-\mathbb{1}) P_{0}\right\}$ is compact. In fact this operator is given by (4.4) with the symbol $c(x, \xi)$,

$$
\begin{aligned}
c(x \cdot \xi)= & (U(x)-\mathbb{1}) \frac{m \beta}{\left[|\xi|^{2}+m^{2}\right]^{1 / 2}}+(U(x)-\mathbb{1}) \frac{1}{\left[|\xi|^{2}+m^{2}\right]^{1 / 2}+|\xi|} \\
& \cdot \frac{\left(-m^{2}\right) \alpha \cdot \xi}{|\xi|\left[|\xi|^{2}+m^{2}\right]^{1 / 2}} .
\end{aligned}
$$

By Lemma 3.3, the symbol $(U(x)-\mathbb{1})\left(1 /\left[|\xi|^{2}+m^{2}\right]^{1 / 2}\right)$ gives rise to a compact operator. Thus

$$
\operatorname{ind}_{P \mathscr{H}} P U P=\operatorname{ind}_{\mathscr{H}}\left\{\mathbb{1}+(U-\mathbb{1}) P_{0}\right\} .
$$

The * algebra generated by $\alpha_{k}=\alpha_{k}^{*}$ and $\beta$ has the unique irreducible representation (up to unitary equivalence). Thus we set

$$
\alpha_{k}=\left(\begin{array}{cc}
\sigma_{k} & 0 \\
0 & -\sigma_{\kappa}
\end{array}\right) \quad \beta=\left(\begin{array}{ll}
0 & 1 \\
1 & 0
\end{array}\right) .
$$

In this representation of Dirac matrices the operator in the right-hand side of (4.29) decouples into two operators and

$$
(4.29)=\operatorname{ind}(\mathbb{1}+(U-\mathbb{1}) \bar{P})+\operatorname{ind}(\mathbb{1}+(U-\mathbb{1})(\mathbb{1}-\bar{P})) \text {, }
$$

where $\bar{P}$ denotes the projection with symbol $(2.9 \mathrm{a})$. The operator $(\mathbb{1}+(U-\mathbb{1}) \bar{P})$ $(\mathbb{1}+(U-\mathbb{1})(\mathbb{1}-\bar{P}))-U=(U-\mathbb{1}) \bar{P} U(\mathbb{1}-\bar{P})$ is compact by the proof of Lemma 3.6. 
This combined with Lemma 3.2 (iii) leads to

$$
\operatorname{ind}_{P \mathscr{H}} P U P=\operatorname{ind}_{\mathscr{H}} U=0
$$

Remark. A non-trivial example of index in (4.2a) is given by

$$
U(x)=\frac{|x|^{2}-1}{|x|^{2}+1}+\frac{-2 i \sigma \cdot x}{|x|^{2}+1} \text { for } G=S U(2) .
$$

By integrating (4.2a), we have $\operatorname{ind}_{P \mathscr{H}} P U^{n} P=n$. (For the detail of the computation, see Sect. 4 of $[10]$.)

\section{Remarks}

We conclude this article by two remarks.

1. As explained in Sect. 1, if $[P, S]$ is of Hilbert-Schmidt class and ind $_{P \mathscr{H}} P S P=n$, the second quantized scattering operator shifts $n$ charges. But we have not tried to estimate the Hilbert-Schmidt norm.

It is plausible that the finiteness of this norm leads automatically to the triviality of index because the integer invariant (e.g. instanton number, monopole charge) appears only when the local gauge transformation enters into the systems, and we know that the local gauge transformation on $R^{3}$ is not unitarily implementable on Fock representations of free fields.

We may of course improve our results by use of more refined methods than the Dyson expansion, but we do believe that the essential mechanism giving rise to the non-trivial index is the same as our results.

We also remark that the index in the massless case studied in this article corresponds to the chiral charge shift while the index for the ordinary charge vanishes in our assumptions by the same reason to the massive case.

In contrast with higher dimensional cases, the index of the scattering operator and the Hilbert-Schmidt norm can be exactly computed in the $1+1$ dimensional massless case. The result is that both chiral and ordinary charge shifts may occur.

2. We now discuss the physical relevance of our technical conditions $A$ and $B$.

First recall that the physical observable quantity is not the connection $A(t, x)$ itself but the curvature $F(t, x)$. So we may claim that the change of the connection yields no physical effects as far as the curvature is fixed.

We consider the curvature $F$ with compact support which is the most easy and physically reasonable case. If the curvature vanishes the potential is a pure gauge, $A_{k}(t, x)=\partial_{k} W(t, x) \cdot W(t, x)^{*}$ for $|x|+|t| \gg 1$ for some $W(t, x)$ in $C^{\infty}\left(\mathbb{R}^{4}, U(N)\right)$.

If the variables $x$ are written by the spherical coordinate $(r, \omega)$,

$$
W(t, x)=W_{t}(r, \omega)
$$

$W$ can be viewed as a smooth family of mappings from $S^{2}$ to $U(N)$ for each $t$. It is known $\pi_{2}(U(N))=0$. As a consequence, we may deform, without change of 
physical contents, $W_{t}$ to $\tilde{W}_{t}$ satisfying

$$
\tilde{W}_{t}(r, \omega)=\mathbb{1} \quad \text { for } r \geqq C, \quad \text { where } C \text { is a constant. }
$$

Thus for the compactly supported curvature $F$ we may deform continuously the potential satisfying Conditions $A$ and (i) of $B$.

The condition (ii) of $B$ is necessary for the unitarity of the scattering operator.

Conclusion. If the curvature is compactly supported, we may deform continuously the connection to fit to Assumptions $A$ and $B$. This deformation gives no physical effects.

\section{References}

1. Araki, H.: Bogoliubov automorphisms and fock representations of canonical anticommutation relations. Preprint R.I.M.S.

2. Baum, P., Douglas, R. G.: $K$ homology and index theory, Proc. Symp. Pure Math. 38, Part 1, $117-173(1982)$

3. Carey, A. L., O'Brien, D. M.: Absence of vacuum polarization in fock space. Lett. Math. Phys. 6, 335-340 (1982)

4. Fedosov, B. V.: Direct proof of the formula for the index of an elliptic system in Euclidean space, Funct. Anal. Appl. 4, 339-341 (1970)

5. Hörmander, L.: The analysis of partial differential operators III. Berlin, Heidelberg, New York: Springer 1985

6. Kumanogo, H.: Pseudo-differential operators Cambridge, MA: M.I.T. Press 1982

7. Matsui, T.: Factoriality and quasi-equivalence of quasifree states for $\mathbb{Z}_{2}$ and $U(1)$ invariant CAR algebras. (To appear)

8. Palmer, J.: Scattering automorphisms of the Dirac field. J. Math. Anal. Appl. 64, 189-215 (1978)

9. Reed, M., Simon, B.: Methods of modern mathematical physics, I. Academic Press New York: 1972

10. Reuter, M., Dittrich, W.: Selected topics in gauge theories. Lecture Notes in Physics, Vol. 244. Berlin, Heidelberg, New York: Springer 1985

11. Ruijsenaars, S.: Gauge invariance and implementability of the $S$-operator for spin 0 and spin $\frac{1}{2}$ particles in time dependent external fields. J. Funct. Anal. 33, 47-57 (1979)

12. Seipp, H. P.: On the $S$-operator for the external field problem of QED, Helv. Phys. Act. 55, 1-28 (1982)

13. Uhlenbeck, K.: The chern classes of sobolev connections. Commun. Math. Phys. 101, 449-457 (1985)

Communicated by H. Araki

Received September 17, 1986; in revised form January 6, 1987 
